\title{
Ulnar nerve compression in the cyclist's hand: two case reports and review of the literature
}

\author{
C. Maimaris FRCS(Ed) and H. G. Zadeh MB BS \\ Accident and Emergency Department, Guy's Hospital, London, UK
}

Compression of the ulnar nerve in the hand is well recognized; over 20 causes have been reported ${ }^{1}$. They can be subdivided into two major groups: compression secondary to space occupying lesions, the commonest being ganglia, and compression secondary to trauma. Traumatic causes include hand lacerations, carpal bone fractures and direct trauma such as in karate ${ }^{2}$.

Ulnar neuropathy also occurs in those who suffer constant or repeated blows to the base of the palm, such as in construction workers and carpenters who use pneumatic drills or other vibrating tools ${ }^{3}$. It can follow sports activities such as video game playing ${ }^{4}$, motor cycling and cycling 5 . Cyclists experience symptoms after prolonged riding 6 . Burke suggested that in cyclists it is an over-use injury ${ }^{7}$.

\section{Ulnar nerve anatomy in the hand (Figure 1)}

The ulnar nerve enters the hand after emerging from beneath the tendon of flexor carpi ulnaris. Here it lies deep to palmar aponeurosis and palmaris brevis, superficial to flexor retinaculum, lateral to pisiform and medial to hook of hamate. In this space, Guyon's canal, it separates into a deep motor branch and a superficial sensory branch. The sensory branch runs over the hypothenar muscles and forms the digital nerves to little finger and ulnar half of the ring finger. The deep motor branch runs deeply in Guyon's canal, between the origins of flexor and abductor digit minimi. Then it disappears under the origin of opponens digiti minimi, grooves the hook of hamate and arches radially leaving the canal. Within this course, it supplies the three hypothenar muscles and later all the interrosseus muscles, adductor pollicis and usually the two ulnar lumbricals.

Ulnar neuropathies in the hand have been classified into five types depending on the level of the lesion: Type I (proximal to the bifurcation, just outside Guyon's canal) to Type V (distal deep palmar branch $)^{8}$. The neurological deficit in each type is as follows: Type I - mixed motor and sensory; Type II pure sensory; Type III - pure motor; Type IV - pure

Address for correspondence: $C$. Maimaris, Accident and Emergency Department, Kent and Canterbury Hospital, Canterbury, CT1 3NG, UK

(C) 1990 Butterworth-Heinemann Ltd 0306-3674/90/040245-02 motor but sparing hypothenar muscles; and Type V distal motor.

\section{Case reports}

\section{Patient 1}

A 47-year-old business man presented to the accident and emergency department complaining of weakness and clumsiness in his left hand for 5 days. His symptoms started after 4 days of cross country cycling over 80 miles. While he was cycling, his left hand was constantly weight bearing on the handlebars but the right hand was frequently off the handlebar when changing gears. Owing to the unevenness of the roads he was getting repeated blows to his hands though the handlebars. On examination of the left hand there was mild clawing of the little finger with marked weakness in the abductor digiti minimi, weakness in abduction and adduction of the fingers and a positive Froment's sign (Figure 2). There was no sensory loss and the radial and median nerves were intact. There was no neurological deficit in the right hand.

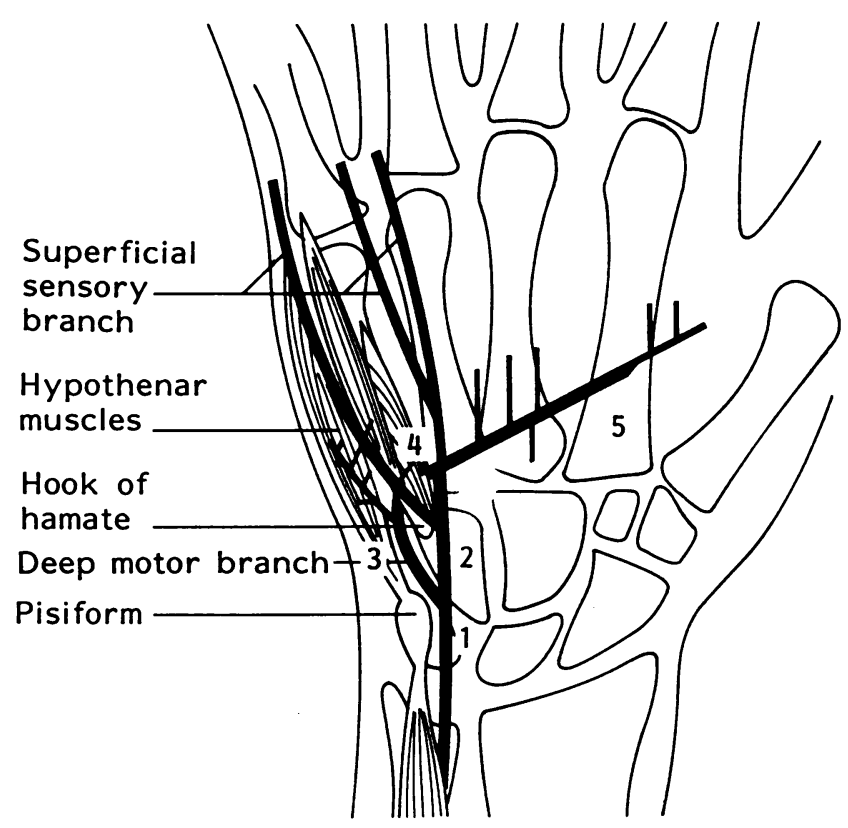

Figure 1. Course of ulnar nerve in the hand demonstrating classification of neuropathies, numbers 1-5 show level of lesion in types I-V neuropathies 


\section{Patient 2}

A 24-year-old dental student undertook a 120 mile bicylce ride in 10 hours the day before attending the accident and emergency department. He cycled from London to Brighton and back, with a 2 hour break in Brighton. He noticed tingling in the left little finger on the return jouney and some weakness in the hand at the end of the ride. There were no symptoms in the right hand. Examination of the left hand showed diminished pin-prick sensation in the left little finger and decreased power of abduction and adduction on the left little finger. Froment's sign was mildly positive. The right hand was normal. Examination 4 days later showed complete recovery of both sensory and motor branches of the ulnar nerve.

\section{Discussion}

The first patient presented with neuropathy of the deep motor branch of the ulnar nerve. The second patient had mixed sensory and motor deficit. In both patients the left ulnar nerve was affected, sparing the right hand. The complete recovery of the neurological deficit implies that the underlying lesion was transient neuropraxia. We think this is due to compression of the nerve by continuous gripping pressure and repeated blows inflicted by the handlebars. The right hand was gripping the handlebars intermittently, so recovered while changing gears.

From the clinico-anatomical presentation, the first patient had pure motor, Type III, ulnar neuropathy. In this type there is compression of the deep branch in the canal of Guyon, affecting the hypothenar and other small muscles of the hand. Clawing is common $^{8}$. The second patient had a Type I (mixed sensory and motor) lesion, in which the compression of the nerve is just proximal to the bifurcation (Figure 1).

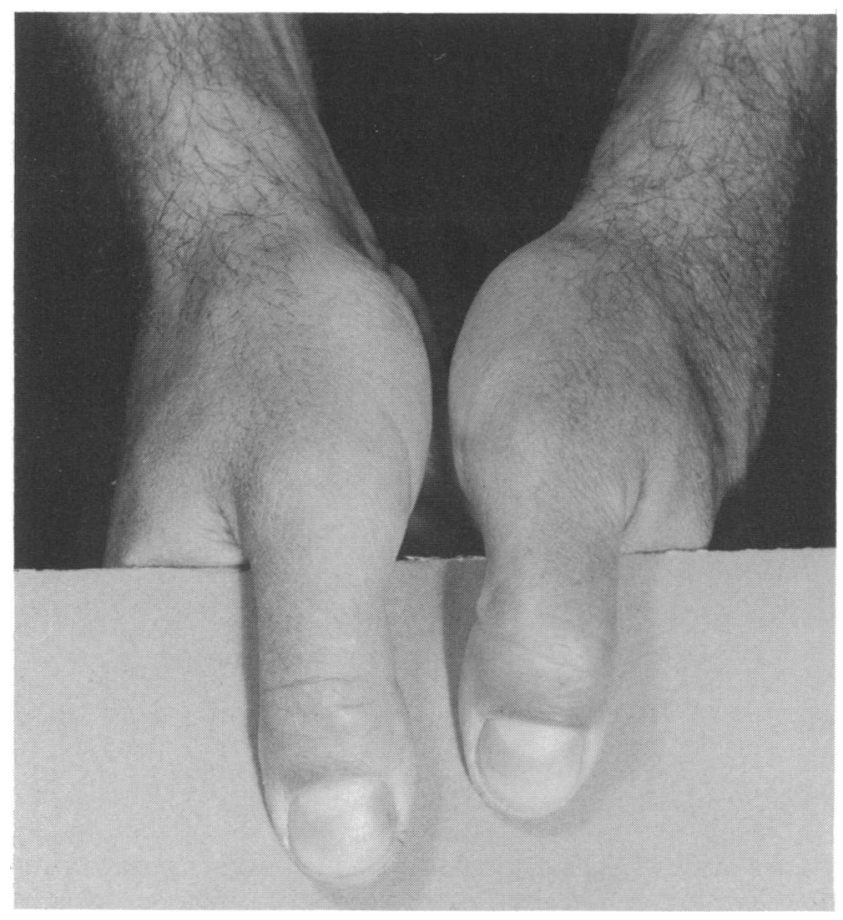

Figure 2. Ulnar nerve palsy, Froment's sign

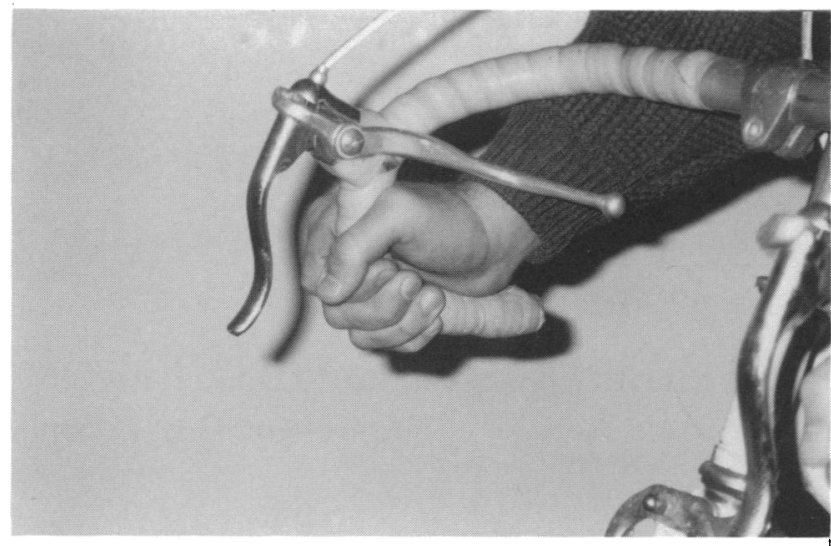

Figure 3. Compression of ulnar nerve by handlebar pressure

It usually occurs just outside Guyon's canal and clawing of the fingers is not a constant finding.

Several factors may contribute to ulnar neuropathy in cyclists. Wearing worn-out gloves or no gloves at all and having unpadded handlebars that transmit vibration to the hands may compress the nerve in the palm. Undue pressure can be caused by prolonged gripping of the drop handle bars especially in the low, forward leaning position (Figure 3). Hence the neuropathy is known as 'handlebar palsy"7.

Both patients were managed conservatively and had a short duration of symptoms. If the symptoms are not relieved, or there is clinical suggestion of space occupying lesion, further investigations are recommended. With prolonged compression the nerve may not recover ${ }^{3}$.

In conclusion, we advise cyclists to wear thick cycling gloves and use bicycles with well padded handlebars. They should frequently change hand positions on the handlebars and thus avoid prolonged gripping, especially during long distance cycling. If symptoms are not relieved by these measures they should stop cycling until improvement occurs.

\section{Acknowledgements}

We would like to thank Major-General N. G. Kirby for allowing us to report on his patients and his advice in preparing this paper.

\section{References}

1 Sharara, K.H. and Nairn, D.S. Metastatic calcification as a cause of ulnar nerve compression at the wrist The Hand 1983, 15 (3), 300-303

2 Helal B., King J. and Grange W. 'Sports Injuries and Their Treatment' Chapman and Hall 1989, 284-285

3 Bakke, J. L. and WOlff, H. G. Occupational pressure neuritis of the deep palmar branch of the ulnar nerve Arch Neur Psych $1948,60,549-53$

4 Friedland, R.P. Video-game palsy New Engl J Med 1984, 311 (1), 58-9

5 Converse, T.A. Cyclists' palsy $N$ Engl J Med 1979, 301 1397-1398

6 Eckman, P.B. Perlstein, G. and Altocchi , P.H. Ulnar neuropathy in bicycle riders Arch Neurol 1975, 32, 130-132

7 Burke, E.D. Ulnar neuropathy in bicyclists Phys Sports Med 1981, 9 (4), 53-56

8 Wu, J.S. et al. Ulnar neuropathy at the wrist: case report and review of literature Arch Phys Med Rehab 1985, 66, 785-89 